\title{
Croton Oil
}

National Cancer Institute

\section{Source}

National Cancer Institute. Croton Oil. NCI Thesaurus. Code C29817.

A yellow to brown colored, thick, foul smelling, very toxic oil obtained from the seeds of a tropical plant, Croton T iglium. Croton oil was used as a drastic purgative agent, but is now mostly used as a standard irritant in pharmacological research. Exposure to this substance causes severe skin irritation and affects the small intestine leading to severe diarrhea. Croton oil is a possible human carcinogen. ( $\mathrm{NCl05)}$ 